\title{
Partitioning within-species variance in behavior to within- and between-population components for understanding evolution
}

\author{
László Zsolt Garamszegi ${ }^{1, *}$ and Anders Pape Møller² \\ ${ }^{1}$ Estación Biológica de Doñana-CSIC, Seville, Spain e-mail: \\ laszlo.garamszegi@ebd.csic.es; ${ }^{2}$ Université Paris-Sud, CNRS, Orsay Cedex, \\ France, e-mail: anders.moller@u-psud.fr \\ *correspondence to LZG: Department of Evolutionary Ecology, Estación \\ Biológica de Doñana-CSIC, c/ Americo Vespucio, 26, 41092, Seville, Spain; \\ Tel: (+34) 954232340 ext. 1111, Fax: (+34) 954621125
}

Statement of authorship: APM collected data, LZG designed the study and performed the statistical analyses, both authors drafted the manuscript

Data accessibility statement: data supporting the results will be archived as a supplementary online material (submitted together with the manuscript)

Running title: Variance components of behavior and evolution

Keywords: ecological plasticity, life history; mixed-models; personality; Pace of Life Syndromes; repeatability; variation in behavior

Type of article: Letters

Word count: $147($ abstract $)+4271$ (main text)

+53 references +1 table +4 figures 


\section{$30 \quad$ Abstract}

31 Phenotypes vary at multiple hierarchical levels, of which the interspecific variance is the primary focus of phylogenetic comparative studies. However, the evolutionary role of particular within-species variance components (betweenpopulation, between- or within-individual variances) remains neglected. Here we partition the variance in an anti-predator behavior, flight initiation distance (FID), and assess how its within- and between-population variance are related to life history, distribution, dispersal and habitat ecology. Although the composition of within-species variance in FID depended on the phylogeny, most variance occurred within populations. When accounting for allometry, densitydependence, uncertainty in the phylogenetic hypothesis, and heterogeneity in data quality, within-population variance was significantly associated with habitat diversity and population size. Between-population variance was a

43 significant predictor of natal dispersal, senescence and habitat diversity. 44 Accordingly, not only species-specific mean values of a behavioral trait, but also

45 its variance within and among populations can shape the evolutionary ecology 46 of species. 


\section{Introduction}

Observations can be summarized as moment statistics such as the first moment, variance, skewness and kurtosis (Wright 1968). Since the days of Fisher (1930), a primary interest in biology focuses on different variance components.

Variances can be decomposed into variance within individuals, which relates to phenotypic plasticity (West-Eberhard 2003; Dingemanse et al. 2010). Variance among individuals is typically what most evolutionary studies target when investigating phenotypic variation in relation to fitness (Williams 1966), but it also has consequences for estimating repeatability, heritability and other quantitative genetic parameters (Falconer \& Mackay 1996). Variance among populations reflects differentiation among populations leading to insipient speciation (Coyne \& Orr 2004). Finally, variance among species and higher taxa reflects evolutionary processes on a phylogenetic scale giving rise to adaptive radiation (Schluter 2000).

Given the different biological relevance of particular variance components, inferences are typically made at a certain hierarchical level, while variances at lower level(s) remain under-appreciated. For example, studies aiming at identifying the proximate or ultimate determinants of phenotypic variation among individuals typically target individual-specific mean trait values and inherently consider within-individual variance to be negligible (Dingemanse et al. 2012). Furthermore, phylogenetic studies often focus on interspecific trait variance requiring species-specific mean trait values with imperceptible withinspecies variance (Ives et al. 2007). However, as phenotypic variation is likely caused by processes operating at multiple hierarchical levels, the focus on a single variance component stands on weak biological foundations. Therefore, understanding why a given phenotype is expressed in a given observation, in a given individual from a given population of a given species necessitates hierarchically structured data, from which the importance of within-individual, between-individual, between-population and between-species components can 
be evaluated in parallel. Although the partitioning of within- and betweenindividual variances based on the use of repeated observations of the same individuals in a mixed-model framework is becoming a common practice (Westneat et al.2014), such appraisals at higher organization levels are scare at best due to data limitation (Albert et al. 2011; Siefert et al. 2015).

Theory and simulation suggest that within-species variance can be a confounding factor when investigating patterns of variance at the among-species level in a phylogenetic context (e.g. Ives et al. 2007; Hansen \& Bartoszek 2012). These concerns arise primarily from the statistical perspective emphasizing that within-species variance can cause bias in the estimation of the evolutionary parameter of interest. Accordingly, within-species variance is an unwanted factor that needs to be accounted for in a phylogenetic comparative study, but it is not of biological interest. From the statistical point of view, it is also irrelevant whether within-species variance emerges because the populations within species are different, because there is remarkable between-individual variance, or because individuals display their phenotypes with high plasticity (Garamszegi 2014). However, species may be different not only in their mean phenotype, but also in how they divide the phenotypic expression of a trait among different hierarchical levels. Therefore, biologically, different withinspecies variance components may play different evolutionary roles.

In a phylogenetic comparative study, we here show that assessing the evolutionary importance of different within-species variance components concordantly can be straightforward for understanding how different selective factors shape highly plastic traits like behaviour on a phylogenetic time scale. For our exemplary analysis, we used extensive data on flight initiation distance (FID), which is a well-studied phenomenon in both the within- and betweenspecies contexts in many animal taxa (Stankowich \& Blumstein 2005). FID is an estimate of risk taking behaviour, and it can be measured as the distance at which an individual animal flees when approached by a potential predator. FID 
107 has been studied mostly in birds and displays substantial variance at different

108 hierarchical levels, with each having biological relevance. At the between-

109 individual level, FID has clear fitness links because individual birds that fall

110 prey to a predator have shorter FID than survivors (Møller 2014). Differences

111 among individuals in FID can partially be explained by the genetic

112 determination of the trait as reflected by studies reporting associations with

113 different genetic markers (Garamszegi et al. 2014, 2015b). Interspecifically, FID

114 correlates with many ecological and evolutionary factors, such as predation

115 pressure (Møller 2008; Møller et al. 2010), morphology (Fernandez-Juricic et al .

116 2006), life history (Blumstein 2006), urbanization (Møller 2010; Carrete \& Tella

117 2011) and plumage vividness (Hensley et al. 2015). Previously, we have

118 analysed means and variances in FID in European birds in an attempt to identify

119 the most important predictors of these moment statistics (Møller \& Garamszegi

120 2012). Both of these were correlated with a number of variables describing

121 habitat selection, breeding range and population size, dispersal, and life history.

122 Although these findings lend support to the evolutionary importance of the

123 within-species variance in FID, it remains elusive which component of within-

124 species variance is responsible for the detected patterns.

125 The objectives of this study, therefore, were to partition variance

126 components and investigate the effects of such partitioning for the evolution of

127 life history characters. We used information on FID for 254 species collected in

128 seven geographically distant localities. From data on almost 10,000 individuals,

129 we calculated site-specific means and variances of FID for each species under

130 the assumption that different sites represent different populations. The species-

131 specific between-population variance was estimated based on the site-specific

132 estimates. This tabulation of data permitted us to investigate the relationship

133 between different variance components and also to estimate their repeatability.

134 Finally, we evaluated how these variance components were related to

135 geographic distribution and life history of different species as reflected by 
136 habitat preference, number of habitats, breeding range, population size, natal

137 dispersal, age at first breeding, survival, longevity and the duration of the 138 breeding season.

140 Material and methods

\section{Observations}

142 APM recorded FID in the field in Denmark, Finland, France, Iceland, Norway,

143 Spain and Ukraine (one site in each country, see Figure 1) during the non-

144 breeding seasons 2006-2016. These data amounted to 9901 observations in total.

145 When an individual bird had been located with a pair of binoculars or with

146 eyesight, APM moved at a normal walking speed towards the individual, while

147 recording the number of steps (which approximately equals the number of

148 meters). The distance at which the individual took flight was recorded as the

149 FID, while the starting distance was likewise recorded (i.e. the experimenter-

150 subject distance when approach began, Blumstein 2003). There was only a weak

151 correlation between FID and starting distance, probably because the observer

152 made efforts to approximate all individuals around 30 meters (GLMM with

153 species as a random effect and locality as a fixed effect and starting distance as a

154 covariate: $F=34.18, \mathrm{df}=1,933.7, P<0.0001$; effect size $\mathrm{r}=0.006)$. Thus we

155 did not consider starting distance in the subsequent analyses. If the individual

156 was positioned in the vegetation, the height above ground was recorded to the

157 nearest meter. APM only recorded FID on days with fine weather conditions and

158 little or no wind or precipitation. FID was estimated as the Euclidian distance,

159 which equals the square-root of the sum of the squared horizontal distance and

160 the squared height above ground level (Blumstein 2006). APM avoided effects

161 of pseudo-replication by only recording a single individual of a given sex, age

162 and species at a given site. Note by measuring individuals only once we actually

163 cannot separate between-individual and within-individual variances, as the

164 detected phenotypic variance within a population is a combination of both 
165 (Westneat et al. 2014). With this shortcoming (see Discussion), resampling by

166 mistake does not lead to additional limitations for the purpose of this study. All

167 FID data analysed here were collected in the same way by the same person

168 implying that there was no scope for among-observer variation.

169 We assume that different sites from different countries represent

170 independent populations. This assumption arises because the data were collected

171 within a radius of $10 \mathrm{~km}$ in each country, and considering that the study

172 organisms are flying birds, this distance likely covers socially interacting

173 individuals. Furthermore, the smallest distance between these sampling sites is

$174 \sim 300 \mathrm{~km}$ (Figure 1), and thus within-site distance is negligible as compared to

175 the between-site distance. However, we cannot exclude that some variance

176 within sites emerges due to habitat heterogeneity, whereby different populations

177 may segregate within sites. To avoid overestimation of the within-population

178 variance, we repeated our analyses based on a stricter definition for populations,

179 in which we considered larger habitat similarity by focusing on observations that

180 correspond to rural environments only. In a similar vein, within-site variance

181 may be biased due to the fact that different sites were sampled in different years.

182 Therefore, we also repeated our analyses after excluding sites, for which data

183 were available for less than three years. The results obtained under these

184 considerations and relying on different subsamples are presented as Supporting

185 Information.

The repeatability of variance components and their relationship

188 To assess the repeatability of within- and between-population variances and

189 investigate their association, we tabulated the data as follows. First, we obtained

190 estimates for the mean and the standard deviation (SD) of the phenotypic

191 observations of FID within each population of each species. The SD of

192 phenotypes was assumed to reflect within-population variance $\left(\mathrm{SD}_{\mathrm{wn}}\right.$ that, given

193 the available data structure, combines between-individual and within-individual 
194 variance). Population-specific mean estimates were used to derive between-

195 population variance in the form of the SD of the means across populations in 196 each species $\left(\mathrm{SD}_{\mathrm{bw}}\right)$, and these were weighted by the underlying sample size (for species, which were sampled in a single country only, such information could not be obtained thus could not be included in the respective analyses). We also calculated the weighted mean of the within-species SDs to obtain speciesspecific estimates for the within-species variance $\left(\mathrm{SD}_{\text {spec }}\right)$. The joint logtransformation of the mean and the variance can only be achieved by approximations (Ives et al. 2007). Therefore, for the analyses relying on the logscale, we first preformed a $\log _{10}$-transformation on the raw individual-specific FID values, then calculated means and SDs over the transformed variables (Garamszegi 2014).

The repeatability of the within-population variance was based on the population-specific estimates. We built a linear mixed model, in which the $\mathrm{SD}_{\mathrm{wn}}$ on the log-scale was used as a response variable and species identity was a random factor. The model also contained a fixed factor for the country of origin, and the population-specific mean of $\log _{10}$-transformed FID as a predictor (to control for the statistical phenomenon that a larger mean is typically associated with a larger variance, Wright 1968). To account for differences in sample size, we weighted each population-specific observation based on the underlying sample size. We used $\log _{10}$-transformed number of observations to avoid overemphasis on species with very large sample sizes (Garamszegi \& Møller 2010). The repeatability of $\mathrm{SD}_{\mathrm{wn}}$ of FID could be estimated from this model by subtracting the appropriate between-species variance component of the trait and 218 relating it to the total variance (Nakagawa \& Schielzeth 2010). The 95\% confidence interval $(95 \% \mathrm{CI})$ was obtained by parametric bootstrap.

To calculate the repeatability of between-population variance, we selected 221 species for which FID estimates were available for at least four localities (20 species). In each of these species, we randomly selected two populations and 
223 calculated the weighted $\mathrm{SD}_{\mathrm{bw}}$ of their respective mean FID (both moments on

224 the log-scale). We also derived such a metric for the remaining populations, and 225 thus we could obtain two parallel estimates for the species-wise between226 population variance. To estimate the repeatability of between-population 227 variance, we estimated the correlation between these two sets of estimates by 228 first controlling for their respective means. We extracted residuals from a 229 regression model that characterises the relationship between the $\log _{10^{-}}$ 230 transformed mean and the $\mathrm{SD}_{\mathrm{bw}}$ of FID. Given that the random selection of 231 populations within species could result in several combinations, we repeated the 232 above process 10,000 times. The mean and the 95\% quantile range of the 233 correlation estimates (that were weighted by sample size) were used to make 234 implication about the $95 \% \mathrm{CI}$ of the repeatability of $\mathrm{SD}_{\mathrm{bw}}$.

\section{Comparative analyses}

237 Data on species-species ecology, life history and distribution originated from 238 different sources mainly from Cramp and Perrins (1977-2002). The description of variables and details of data extraction are given in Møller and Garamszegi

240 (2012). Briefly, body mass reflected the mean body mass of adults during 241 breeding. Habitat scores of 1,2 and 3 reflected grassland, shrub and trees, respectively. The number of habitat types on which the given species occurs during reproduction was used as a measure of habitat exploitation. Distribution was the area in $\mathrm{km}^{2}$ of the breeding range. Population size was the number of breeding pairs in the Western Plaearctic listed by Burfield and van Bommel (2004). Age at first reproduction was the mean age at first reproduction in years. Natal dispersal was the geometric mean distance between natal site and subsequent breeding site according to Paradis et al. (1998). Fecundity was clutch size multiplied by the maximum number of clutches per year. Survival was the adult survival rate. Longevity was the maximum longevity in years according to 
252 number of 10 day periods with reproduction. Study effort was the number of 253 publications for each species as listed by Web of Science. The variables were $254 \log _{10}$-transformed except survival rate that was square-root arcsine transformed. 255 The comparative dataset is provided as an electronic supplementary material.

To control for effect due to common descent in our statistical models we used information on the phylogenetic relationships of species from Jetz et al. (2012). We downloaded 100 trees representing a set of equally likely hypotheses for the underlying phylogeny (see one example in Figure 2). Variance across these trees reflects the uncertainty in the phylogenetic hypothesis, for which we accounted by multimodel inference (Garamszegi \& Mundry 2014). Accordingly, we fitted a Phylogenetic Generalized Least Squares (PGLS) model on each tree, in which we forced the correlation structure of residuals to follow the phylogenetic relatedness of species as described for the underlying phylogenetic tree (Paradis 2011). In these models, we allowed the phylogenetic scaling factor, $\lambda$ to take its maximum-likelihood value (Freckleton et al. 2002). This parameter can vary from 0 reflecting no phylogenetic signal in the residuals to +1 indicating strong phylogenetic constraints. We applied a model averaging technique over the 100 models corresponding to different trees to derive the parameter of interest (i.e. regression slope or $\lambda$ ).

Sample sizes varied among species, which can raise issues about heterogonous data quality (Garamszegi \& Møller 2010). Accordingly, we weighted each observation in the phylogenetic models based on the underlying within-species number of observations of FID following procedures from Garamszegi (2014). We used $\log _{10}$-transformed weights, which were strongly positively correlated with the number of populations from which the data originated $(r=0.701, N=254, P<0.001)$ and also with the number of years when the species were sampled $(r=0.844, N=254, P<0.001)$.

We constructed PGLS models to test for the mean/variance relationship on both non-transformed and log-scale. The latter models relied on the mean of 
$281 \log _{10}$-tranformed FID as predictor, and one of the variance estimates (either $282 \mathrm{SD}_{\mathrm{wn}}$ or $\mathrm{SD}_{\mathrm{bw}}$ of $\log _{10}$-tranformed FID) as response. $\log _{10}$-tranformed SD 283 estimates were entered into a PGLS model investigating whether they vary 284 independently from each other. To assess the relationship between variance 285 components and the considered species-specific traits, we built multivariate 286 PGLSs, in which we accounted for different potentially confounding factors 287 under the following particular considerations.

288 We expected that species would be using more habitats if their breeding 289 range was larger because a larger range would imply a greater opportunity to 290 encounter different habitats. For the analysis of population size we included 291 range size because a larger range would imply more individuals. For natal 292 dispersal we included population size and range size because a larger population 293 size for a given area would increase density-dependent natal dispersal. For age 294 at first reproduction we included population size and range size because higher 295 density favours early start of reproduction. For survival and fecundity, we 296 included population size and breeding range to account for density-dependence.

297 For longevity we included survival rate because higher survival increases 298 longevity. In this model, we also included population size and breeding range to 299 account for density-dependence, as well as research effort in terms of total 300 number of recoveries of banded birds (as well-sampled species are more likely 301 to be found to have older individuals). Each model included body mass to 302 account for any allometry effects.

\section{Results}

Partitioning variance components of FID

The repeatability of $\mathrm{SD}_{\mathrm{wn}}$ was 0.603 (when we weighted each populationspecific observation based on the underlying sample size, we obtained a similar estimate: $\mathrm{R}=0.581$ ). The associated $95 \% \mathrm{CI}$ yielded a range that was above zero ( 0.482 to 0.707$)$, and it could thus be considered statistically significant. 
310 The mean estimate of the repeatability of $\mathrm{SD}_{\mathrm{bw}}$ was smaller $(R=0.261)$ and

311 corresponded to a $95 \%$ CI (-0.063 to 0.616$)$ that could not be statistically

312 distinguished from zero, probably due to the small sample size.

313 There were 84 species for which we had estimates for both the within-

314 and between-population variance components. A pairwise comparison revealed

315 that $\mathrm{SD}_{\mathrm{wn}}$ was consistently larger than $\mathrm{SD}_{\mathrm{bw}}(t=5.978, \mathrm{df}=83, P<0.001)$. In

316 terms of variances, on average, the within-population variance in FID was

$31774.13 \%$ (s.e. $=3.10 \%$ ) of the total within-species variance, and the remaining

$31825.87 \%($ s.e. $=2.95 \%)$ could be attributed to the between-population variance.

319 However, this partitioning was not homogeneously expressed across the

320 phylogenetic tree, as the proportional distribution of the two components varied

321 among species depending on their phylogenetic positions (the weighted PGLS

322 estimate of the phylogenetic signal of $\log _{10}$-transformed $\mathrm{SD}_{\mathrm{wn}}$ minus $\log _{10^{-}}$

323 transformed $\mathrm{SD}_{\text {spec }}$, reflecting within-population variance relative to the total

324 variance was $\lambda=0.560$, which was associated with a $95 \% \mathrm{CI}=0.515$ to 0.617

325 across trees; Figure 2).

326 There was a strong positive relationship between mean FID and estimates

327 of variance on the non-transformed scale $\left(\mathrm{SD}_{\mathrm{wn}}:\right.$ beta $=0.258,95 \% \mathrm{CI}=0.211$

328 to $0.306 ; \mathrm{SD}_{\mathrm{bw}}$ : beta $=0.161,95 \% \mathrm{CI}=0.062$ to 0.261 ). However, these

329 relationships were not present on the log-scale $\left(\mathrm{SD}_{\mathrm{wn}}\right.$ : beta $=-0.024,95 \% \mathrm{CI}=-$

3300.063 to $0.014 ; \mathrm{SD}_{\mathrm{bw}}$ : beta $=-0.006,95 \% \mathrm{CI}=-0.081$ to 0.069 , Figure $3 \mathrm{~A}$ and

331 B), and thus we did not consider the mean/variance relationships further in

332 analyses on the log-scale. We detected no significant association between $\mathrm{SD}_{\mathrm{wn}}$

333 and $\mathrm{SD}_{\mathrm{bw}}$ (beta $=0.188,95 \% \mathrm{CI}=-0.124$ to 0.499 , Figure $3 \mathrm{C}$ ) indicating that

334 the two estimates were statistically independent.

336 Variance components of FID and distribution, dispersal and life history

337 The results of the multi-predictor models accounting for different potentially

338 confounding factors are shown in Table 1. We repeated the same sets of 
analyses by using different subsets of the data, which were compiled under stricter criteria for habitat homogeneity within sites and balanced sampling effort, respectively. These supplementary analyses yielded somewhat different patterns (Tables S1 and S2) than the outcomes relying on the full data. Hence we point to those effects only that are robust and consistently appear across the different control approaches. There was concordance in that $\mathrm{SD}_{\mathrm{bw}}$ was a positive predictor of natal dispersal, and explained more than $10 \%$ in interspecific variance of the trait (Figure $4 \mathrm{~A}$ ). With a similar effect size, $\mathrm{SD}_{\mathrm{bw}}$ was negatively associated with longevity (Figure $4 \mathrm{~B}$ ). Both $\mathrm{SD}_{\mathrm{wn}}$ and $\mathrm{SD}_{\mathrm{bw}}$, independently accounted for $\sim 5 \%$ of variance in the number of habitats (Figures $4 \mathrm{C}$ and $4 \mathrm{D}$ ). Noteworthy, $\mathrm{SD}_{\mathrm{bw}}$ was strongly and positively associated with population size when using the full data (Table 2), but this relationship was considerably weakened when we restricted our analysis to observations originating from similar habitats (Table S1). We also detected that at least $\sim 10 \%$ of the variance in population size and the duration of breeding season can be attributed to mean FID (Table 1).

\section{Discussion}

The main findings of this study were the following. First, variation in antipredator behavior as reflected by $\mathrm{SD}_{\mathrm{wn}}$ and $\mathrm{SD}_{\mathrm{bw}}$ of FID differed significantly, as the within-population component was consistently larger than the betweenpopulation component, and this was coupled with larger repeatability. Second, the composition of within-species variance depended on the phylogenetic relationships of species. Finally, $\mathrm{SD}_{\mathrm{wn}}$ and $\mathrm{SD}_{\mathrm{bw}}$ of FID differed in their ability to predict species-specific characteristics of life history, distribution, dispersal and habitat ecology. These findings are important because they show that different components of variance capture different biological mechanisms.

Most comparative studies focusing on interspecific variance neglect the importance of within-species variance in the studied trait (Garamszegi \& Møller 
368 2010). In our analyses focusing on the within-species variance of a plastic

369 behavioral trait, we demonstrated that this component can respond to selective 370 mechanisms (Møller \& Garamszegi 2012 ; this study). Our previous analyses 371 revealed that habitat use, range size, population size and several life history 372 traits are associated with $\mathrm{SD}_{\text {spec }}$ of FID. The interpretation of these results was 373 that species that sustain a greater diversity of anti-predator behavior can cope 374 more efficiently with unpredictable environmental conditions allowing them to 375 exploit more habitats, disperse over larger distribution areas, establish higher 376 population densities and adopt fast reproductive strategies. Our current appraisal 377 is mostly in line with this suggestion, even if minor differences in the results 378 appear to be due to dissimilarity in methodology and sample size. The current 379 study adds the important amendment that different evolutionary roles apply to 380 different components of the within-species variance.

381 Although evolutionary processes can concordantly mediate both 382 components of within-species variance, they can also have different 383 consequences at different levels. For example, diversifying selection would shift 384 population-specific means in opposite directions thereby increasing betweenpopulation variances, while leaving within-population variances unaffected or even decreased (Freeman \& Herron 2007; Møller 2008). On the other hand, diversifying or disruptive selection can also occur within populations, which would in turn increase within-population variance while eroding amongpopulation variance. If these selection mechanisms at different hierarchical levels are mediated by different ecological factors, between-population and within-population variances will be decoupled. Our results showing that $\mathrm{SD}_{\mathrm{wn}}$ and $\mathrm{SD}_{\mathrm{bw}}$ of FID are independent, characterized by different repeatability and have different predictive value support that the two components are responsive 394 to different selective forces. population differentiation and ecological plasticity via gene flow (Johnson \& 
397 Gaines 1990). Long-distance dispersal is an important mediator of processes 398 whereby organisms find and successfully colonize novel environments (Shaw 399 1995; Owens et al. 1999). Accordingly, dispersal ability can be hypothesized to 400 either have a homogenizing effect on populations or generate population 401 divergence through improved invasion success (Belliure et al. 2000). Our 402 findings support the second hypothesis, as they show that longer natal dispersal 403 is coupled with higher variance among populations. Furthermore, it is also 404 plausible that longer natal dispersal favors colonizer individuals to settle in more 405 diverse habitats, which would in turn increase variance in behaviors (Riechert \& 406 Hall 2000). The relationship between the number of habitats and the variance 407 components of FID is in line with this scenario.

$408 \quad$ Pace of Life Syndromes (POLS) arise as a consequence of underlying 409 effects of physiology and life history being responsible for behavior (Réale et al. 410 2010). Such POLS are mediated by life history trade-offs, by which individuals 411 or species prioritize future over current reproduction (Roff 2001), and this can 412 have consequences for risk-taking behavior (Wolf et al.2007). At the inter413 specific level, fast-lived species reproduce at an earlier age, have longer 414 breeding seasons, higher annual fecundity and they senesce faster than slow415 lived species (Reynolds 2003). Suites of behavior as those linked to predation 416 (or parasitism) can be considered important elements of such POLS axes, 417 because organisms should be sensitive to predation (or parasitism) risk 418 depending on their residual reproductive value (Wolf et al. 2007). Here we have 419 made extensive analyses of one measure of anti-predator behavior, and shown 420 that species adopting fast reproductive strategies (i.e. have shorter lifespan) also 421 maintain higher between-population variance in the trait. Therefore, in addition 422 to mean behavior their variance components should also be considered for the 423 evolution of POLS and determine ecological plasticity. Accordingly, 424 ecologically plastic species exploit more habitats and can disperse over distant 425 areas partly because maintaining larger variation in behavior allows them to 
cope effectively with unpredictable environments.

Intraspecific phenotypic variation could emerge due to phenotypic plasticity or true between-individual differences in behavior (Dingemanse et al. 2010; Westneat et al. 2014). Unfortunately, with the available data we were unable to decompose variance below the population level. Studies on different species show great diversity in how FID can vary within individuals, as the trait can depict very high (Carrete \& Tella 2010), moderate (Keyel et al. 2012; Møller 2014) or low (Garamszegi et al. 2015a) repeatability. Therefore, we cannot exclude that some amount of the within-population variance originates from within-individual plasticity, at least in some species. Furthermore, results in association with between-population variance should be interpreted with care. Accordingly, differences in the population-specific mean estimates of FID can arise because individuals from different populations express their phenotypes from different parts of the same reaction norms, or because the betweenindividual distribution of trait expression is shifted between populations due to sorting or selection (Schlichting \& Pigliucci 1998; Laurila et al. 2002; Edge et al. 2013). Therefore, future studies are needed from which the evolutionary role of within-individual variance could be assessed.

In conclusion, we have shown that different components of variance in behavior display patterns at the interspecific level that can be the signature of adaptive mechanisms and affect variance components independent of the species-specific mean trait expression. These findings suggest that different variance components of behavior constitute evolved mechanisms that link the variance in behavior within individuals, among individuals and among populations. Therefore, the comparative analyses of within-population and between-population variances of phenotypic traits offer promising avenues for understanding the evolution of such traits. 
455 LZG was supported by funds from The Ministry of Economy and

456 Competitiveness (Spain) (CGL2015-70639-P) and The National Research,

457 Development and Innovation Office (Hungary) (K-115970).

\section{References}

4601.

461 Albert, C.H., Grassein, F., Schurr, F.M., Vieilledent, G. \& Violle, C. (2011). When and how should intraspecific variability be considered in traitbased plant ecology? Perspect Plant Ecol Evol Syst, 13, 217-225.

2.

Belliure, J., Sorci, G., Møller, A.P. \& Clobert, J. (2000). Dispersal distances predict subspecies richness in birds. J Evol Biol, 13, 480-487.

Blumstein, D.T. (2003). Flight-initiation distance in birds is dependent on intruder starting distance. J Wildl Manage, 67, 852-857.

4.

Blumstein, D.T. (2006). Developing an evolutionary ecology of fear: How life history and natural history traits affect disturbance tolerance in birds. Anim Behav, 71, 389-399.

5.

Burfield, I. \& van Bommel, F. (2004). Birds in Europe. BirdLife International, 6.

Carrete, M. \& Tella, J.L. (2010). Individual consistency in flight initiation distances in burrowing owls: A new hypothesis on disturbance-induced 7. habitat selection. Biol Lett, 6, 167-170.

Carrete, M. \& Tella, J.L. (2011). Inter-individual variability in fear of humans and relative brain size are related to contemporary urban invasions in 8. birds. PLOS ONE, 6, e18859.

Coyne, J.A. \& Orr, H.A. (2004). Speciation. Sinauer, Sunderland, MA. 9.

Cramp, S. \& Perrins, C.M. (1977-2002). The birds of the Western Palearctic. 10. Oxford University Press Oxford.

Dingemanse, N.J., Dochtermann, N.A. \& Nakagawa, S. (2012). Defining behavioural syndromes and the role of "syndrome deviation" in understanding their evolution. Behav Ecol Sociobiol, 66, 1543-1548. 11. 
Dingemanse, N.J., Kazem, A.J.N., Reale, D. \& Wright, J. (2010). Behavioural reaction norms: animal personality meets individual plasticity. Trends Ecol Evol, 25, 81-89.

12.

Edge, C.B., Thompson, D.G. \& Houlahan, J.E. (2013). Differences in the Phenotypic Mean and Variance Between Two Geographically Separated Populations of Wood Frog (Lithobates sylvaticus). Evol Biol, 40, 276287.

\section{3.}

Falconer, D.S. \& Mackay, T.F.C. (1996). Introduction to quantitative genetics. 3rd edn. Longman, Harlow.

14.

Fernandez-Juricic, E., Blumstein, D.T., Abrica, G., Manriquez, L., Adams, L.B., Adams, R. et al. (2006). Relationships of anti-predator escape and postescape responses with body mass and morphology: A comparative avian study. Evol Ecol Res, 8, 731-752.

15.

Fisher, R.A. (1930). The genetical theory of natural selection. Clarendon Press, Oxford, UK.

16.

Freckleton, R.P., Harvey, P.H. \& Pagel, M. (2002). Phylogenetic analysis and 17.

comparative data: A test and review of evidence. Am Nat, 160, 712-726.

Freeman, S. \& Herron, J.C. (2007). Evolutionary analysis. Benjamin 18. Cummings, San Francisco, CA.

Garamszegi, L.Z. (2014). Uncertainties due to within-species variation in comparative studies: measurement errors and statistical weights. In: Modern phylogenetic comparative methods and their application in evolutionary biology: Concepts and practice (ed. Garamszegi, LZ). 19. Springer Heidelberg, Germany, pp. 157-199.

Garamszegi, L.Z., Markó, G., Szász, E., Zsebők, S., Azcárate, M., Herczeg, G. et al. (2015a). Among-year variation in the repeatability, within- and between-individual, and phenotypic correlations of behaviors in a natural 20. population. Behav Ecol Sociobiol, 69, 2005-2017.

Garamszegi, L.Z. \& Møller, A.P. (2010). Effects of sample size and intraspecific variation in phylogenetic comparative studies: A metaanalytic review. Biol Rev, 85, 797-805.

21.

Garamszegi, L.Z., Mueller, J.C., Markó, G., Szász, E., Zsebők, S., Herczeg, G. et al. (2014). The relationship between DRD4 polymorphism and 
phenotypic correlations of behaviors in the collared flycatcher. Ecol Evol, 4, 1466-1479.

22.

Garamszegi, L.Z. \& Mundry, R. (2014). Multimodel-inference in comparative analyses. In: Modern phylogenetic comparative methods and their application in evolutionary biology: Concepts and practice (ed. 23. Garamszegi, L.Z.). Springer, Heidelberg, Germany, pp. 305-331.

Garamszegi, L.Z., Zagalska-Neubauer, M., Canal, D., Markó, G., Szász, E., Zsebők, S. et al. (2015b). Malaria parasites, immune challenge, MHC variability, and predator avoidance in a passerine bird. Behav Ecol, 26, 1292-1302.

24.

Hansen, T.F. \& Bartoszek, K. (2012). Interpreting the evolutionary regression: the interplay between observational and biological errors in phylogenetic 25. comparative studies. Syst Biol, 61, 413-425.

Hensley, N.M., Drury, J.P., Garland, T. \& Blumstein, D.T. (2015). Vivid birds do not initiate flight sooner despite their potential conspicuousness. Curr Zool, 61, 773-780.

26.

Ives, A.R., Midford, P.E. \& Garland, T. (2007). Within-species variation and measurement error in phylogenetic comparative methods. Syst Biol, 56, 252-270.

27.

Jetz, W., Thomas, G.H., Joy, J.B., Hartmann, K. \& Mooers, A.O. (2012). The 28. global diversity of birds in space and time. Nature, 491, 444-448.

Johnson, M.L. \& Gaines, M.S. (1990). Evolution of dispersal. Theoretical models and empirical tests using birds and mammals. Annu Rev Ecol Syst, 21, 449-480.

29.

Keyel, A.C., Peck, D.T. \& Reed, J.M. (2012). No Evidence for Individual Assortment by Temperament Relative to Patch Area or Patch Openness in the Bobolink. The Condor, 114, 212-218.

30.

Laurila, A., Karttunen, S. \& Merilä, J. (2002). Adaptive phenotypic plasticity and genetics of larval life histories in two Rana temporaria populations. Evolution, 56, 617-627.

31.

Møller, A.P. (2008). Flight distance of urban birds, predation and selection for 32. urban life. Behav Ecol Sociobiol, 63, 63-75. 
581

582

583

584

585

586

587

588

589

590

591

592

593

594

595

596

597

598

599

600

601

602

603

604

605

606

607

608

609

610

611

612

613

614

615

616

617

618

619

620

621

622

Møller, A.P. (2010). Interspecific variation in fear responses predicts urbanization in birds. Behav Ecol, 21, 3653-3671.

33.

Møller, A.P. (2014). Life history, predation and flight initiation distance in a 34. migratory bird. J Evol Biol, 27, 1105-1113.

Møller, A.P., Erritzøe, J. \& Nielsen, J.T. (2010). Causes of interspecific variation in susceptibility to cat predation on birds. Chinese Birds, 1, 97111.

35.

Møller, A.P. \& Garamszegi, L.Z. (2012). Between individual variation in risk taking behavior and its life history consequences. Behav Ecol, 23, 843853.

36.

Nakagawa, S. \& Schielzeth, H. (2010). Repeatability for Gaussian and non37.

Gaussian data: A practical guide for biologists. Biol Rev, 85, 935-956.

Owens, I.P.F., Bennett, P.M. \& Harvey, P.H. (1999). Species richness among birds: Body size, life history, sexual selection or ecology? Proc $R$ Soc Lond, Ser B, 266, 933-939.

38.

Paradis, E. (2011). Analysis of phylogenetics and evolution with R. 2 edn. Springer, Berlin, Germany.

39.

Paradis, E., Baillie, S.R., Sutherland, W.J. \& Gregory, R.D. (1998). Patterns of natal and breeding dispersal in birds. J Anim Ecol, 67, 518-536. 40.

Réale, D., Garant, D., Humphries, M.M., Bergeron, P., Careau, V.\& Montiglio, P.O. (2010). Personality and the emergence of the pace-of-life syndrome concept at the population level. Philos Trans $R$ Soc B-Biol Sci, 365, 40514063 .

41.

Reynolds, J.D. (2003). Life histories and extinction risk. In: Macroecology: Concepts and consequences (eds. Blackburn, T.M. \& Gaston, K.J.). Blackwell, Oxford, UK pp. 195-217.

42.

Riechert, S.E. \& Hall, R.F. (2000). Local population success in heterogeneous habitats: reciprocal transplant experiments completed on a desert spider. $J$ Evol Biol, 13, 541-550.

43.

Roff, D.A. (2001). Life history evolution. Sinauer, Sunderland, Mass. 44. 
623

624

625

626

627

628

629

630

631

632

633

634

635

636

637

638

639

640

641

642

643

644

645

646

647

648

649

650

651

652

653

654

655

656

657

Schlichting, C.D. \& Pigliucci, M. (1998). Phenotypic evolution: A reaction norm perspective. Sinauer, Sunderland, MA. 45.

Schluter, D. (2000). The ecology of adaptive radiation. Oxford University Press, Oxford, UK.

46.

Shaw, M.W. (1995). Simulation of population expansion and spatial pattern when individual dispersal distributions do not decline exponentially with distance. Proc R Soc B-Biol Sci, 259, 243-248.

47.

Siefert, A., Violle, C., Chalmandrier, L., Albert, C.H., Taudiere, A., Fajardo, A. et al. (2015). A global meta-analysis of the relative extent of intraspecific trait variation in plant communities. Ecol Lett, 18, 1406-1419.

48.

Stankowich, T. \& Blumstein, D.T. (2005). Fear in animals: a meta-analysis and 49.

West-Eberhard, M.J. (2003). Developmental plasticity and evolution. Oxford 50.

Westneat, D.F., Wright, J. \& Dingemanse, N.J. (2014). The biology hidden inside residual within-individual phenotypic variation. Biol Rev, 89, DOI: 10.1111/brv.12131.

51.

Williams, G.C. (1966). Adaptation and natural selection. Princeton University Press, Princeton, NJ.

52.

Wolf, M., van Doorn, G.S., Leimar, O. \& Weissing, F.J. (2007). Life-history trade-offs favour the evolution of animal personalities. Nature, 447, 581584 .

53.

Wright, S. (1968). Evolution and the genetics of populations. Vol. 1. Genetic and biometric foundations. University of Chicago Press, Chicago, IL. 
658 Table 1.

659 Means and different variance components for FID in relation to variables

660 describing species-specific ecology, distribution and life history. Estimates of

661 regression slopes were given by phylogenetic models that were averaged across

662 available phylogenetic trees and that were weighted by underlying sample sizes

663 (number of individuals). Each model included the mean and the two estimates of

664 SD as predictors together with a specific set of confounders (see footnotes for

665 details). For each model we provide the underling sample size in terms of the

666 number of species, and the $95 \% \mathrm{CI}$ of the phylogenetic scaling factor $\lambda$ as

667 estimated across trees. For illustrative purposes, slopes and 95\% CI that differed

668 significantly from zero are underlined (for interpretations see text).

669

\begin{tabular}{|c|c|c|c|c|c|c|}
\hline \multirow[t]{2}{*}{$\begin{array}{l}\text { Response } \\
\text { variable }\end{array}$} & \multicolumn{2}{|c|}{ Mean FID } & \multicolumn{2}{|c|}{$\begin{array}{c}\text { Within-population SD in } \\
\text { FID }\end{array}$} & \multicolumn{2}{|c|}{$\begin{array}{c}\text { Between-population SD } \\
\text { in FID }\end{array}$} \\
\hline & slope & $\begin{array}{c}95 \% C I \\
\text { (upper/lower) }\end{array}$ & slope & $\begin{array}{c}95 \% C I \\
\text { (upper/lower) }\end{array}$ & slope & $\begin{array}{c}95 \% C I \\
\text { (upper/lower) }\end{array}$ \\
\hline $\begin{array}{l}\text { Habitat }^{\mathrm{A}} \\
\mathrm{N}=77 \\
\lambda_{95}=1 / 1\end{array}$ & 0.310 & $-0.362 / 0.982$ & 3.119 & $-0.015 / 6.253$ & -1.161 & $-3.146 / 0.824$ \\
\hline $\begin{array}{l}\text { Number of } \\
\text { habitats }{ }^{A, B} \\
N=82 \\
\lambda_{95}=0 / 0\end{array}$ & $\underline{0.278}$ & $\underline{0.014 / 0.542}$ & 0.947 & $-0.004 / 1.897$ & $\underline{0.892}$ & $\underline{0.049 / 1.736}$ \\
\hline $\begin{array}{l}\text { Distribution } \\
\text { area }^{\mathrm{A}} \\
\mathrm{N}=83 \\
\lambda_{95}=0 / 0\end{array}$ & -0.270 & $-0.582 / 0.041$ & 0.527 & $-0.623 / 1.677$ & 0.164 & $-0.865 / 1.194$ \\
\hline $\begin{array}{l}\text { Population } \\
\text { size }^{\mathrm{A}, \mathrm{B}}\end{array}$ & -1.135 & $-1.733 /-0.537$ & 3.349 & $\underline{1.123 / 5.576}$ & -1.377 & $-3.155 / 0.401$ \\
\hline $\begin{array}{l}N=83 \\
\lambda_{95}=\end{array}$ & & & & & & \\
\hline
\end{tabular}


$0.366 / 0.431$

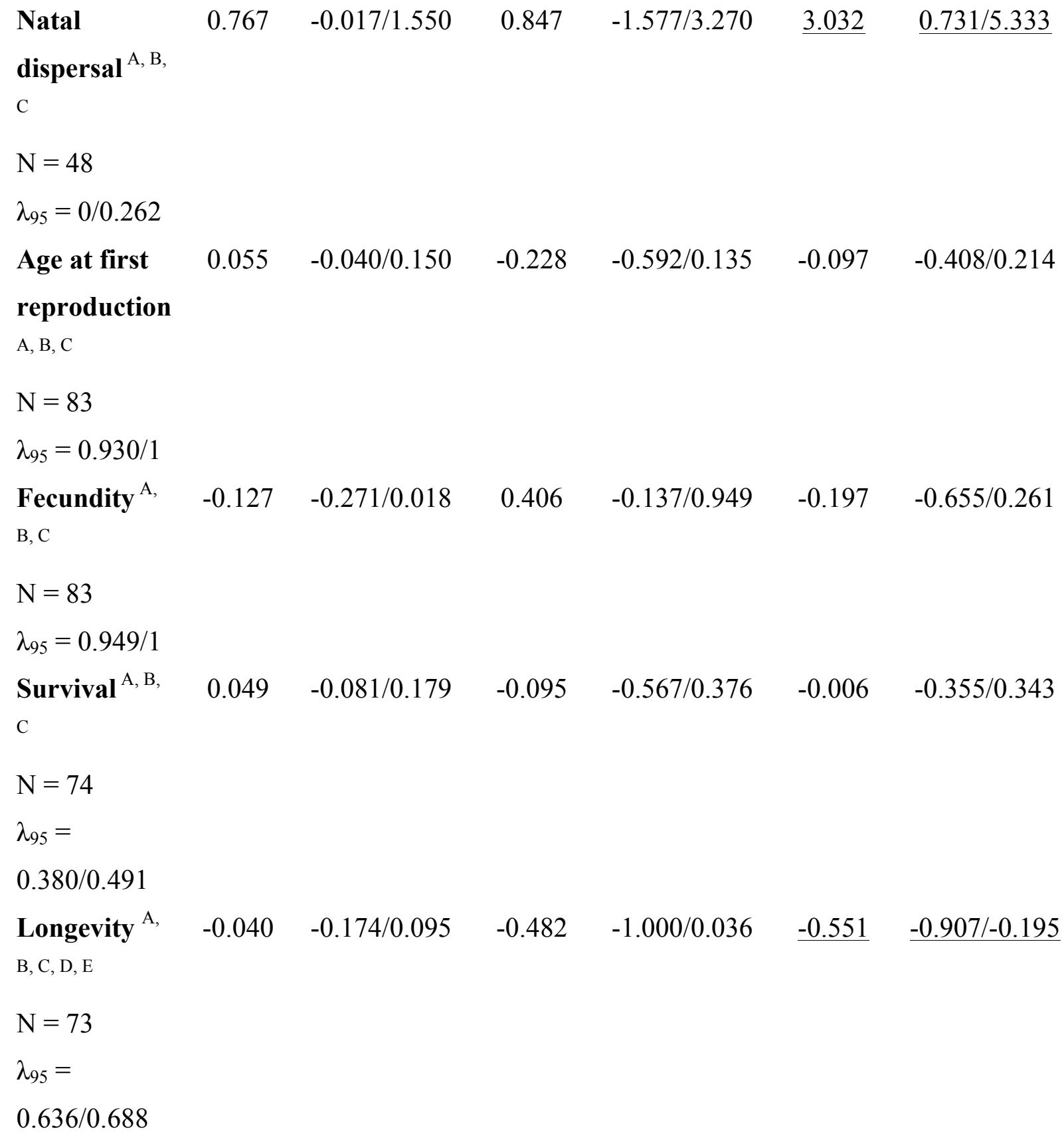

$\begin{array}{lllllll}\text { Duration of } \quad \underline{-0.295} & \underline{-0.442 /-0.148} & 0.444 & -0.016 / 0.904 & 0.239 & -0.186 / 0.664\end{array}$ the breeding

$\operatorname{season}^{A}$

$\mathrm{N}=83$

$\lambda_{95}=0.682 / 1$

670 Variables controlled for in the models: ${ }^{\mathrm{A}}$ : body mass, ${ }^{\mathrm{B}}$ : distribution area, ${ }^{\mathrm{C}}$ : population size, $671{ }^{\text {D: }}$ survival; ${ }^{\text {E}}$ : research effort 
674 Legends to figures

675

676 Figure 1. A map of sampling sites in Europe, where information on FID were 677 obtained to estimate within- and between-population variance of the trait. Data 678 were collected within a radius of $10 \mathrm{~km}$ in each site.

679

680 Figure 2. A phylogenetic hypothesis of bird species (taken from Jetz et al. 681 2012) with information on within- and between-population variance in FID. Pie 682 charts at the tip pf labels show estimated composition of within-species 683 variances (black: between-population, white: within-population variance).

Figure 3. (A-D) The relationship between species-specific estimates of mean FID (flight initiation distance) and its SD reflecting two different components of the within-species variance when using untransformed data $(\mathbf{A}, \mathbf{B})$ and on a logarithmic scale $(\mathbf{C}, \mathbf{D})$. (E) The relationship between $\mathrm{SD}_{\mathrm{wn}}$ and $\mathrm{SD}_{\mathrm{bw}}$ of FID. Regression slopes (black lines) were estimated from phylogenetic models that were averaged across the available phylogenetic trees (grey lines are for particular trees) and that were weighted by the underlying sample size (number of individuals). Model averaged slopes shown with thin dashed lines represent

Figure 4. Different variance components (as estimated by $\mathrm{SD}_{\mathrm{wn}}$ and $\mathrm{SD}_{\mathrm{bw}}$ ) in FID in relation to (A) natal dispersal, (B) longevity and (C-D) number of 697 habitats. Regression slopes (black lines) were obtained from phylogenetic 698 models that were averaged across available phylogenetic trees (grey lines are for 699 particular trees) and that were weighted by the underlying sample size (number 700 of individuals). Slopes and data (in the form of residuals) were extracted from 701 the respective multi-predictor PGLS model that included potentially confounding factors (Table 1). The size of the points is proportional to the 
703 underlining sample size (number of individuals that was used as a weight factor 704 in the statistical models). 

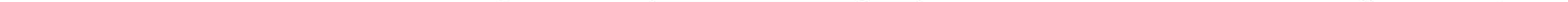


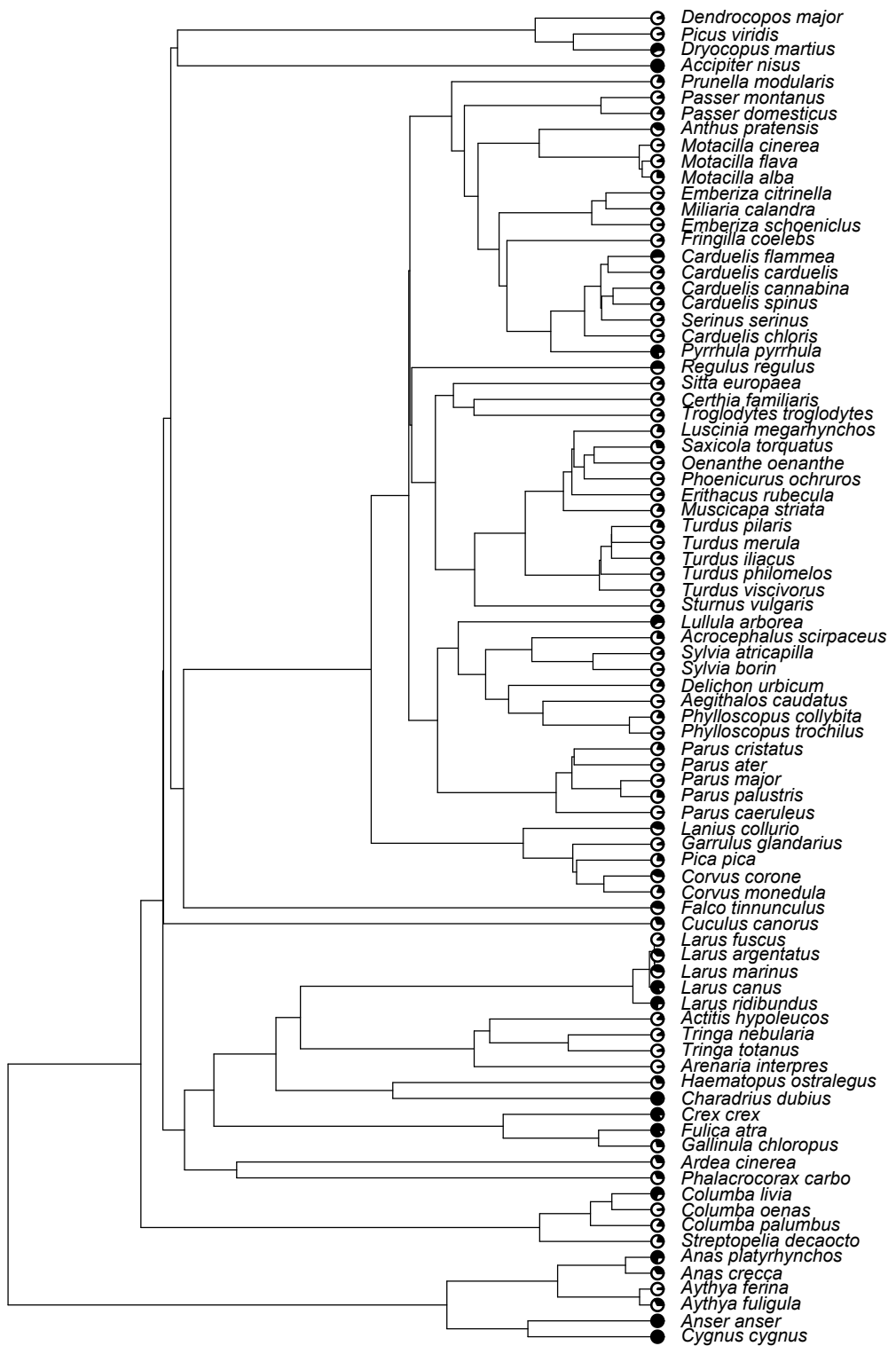



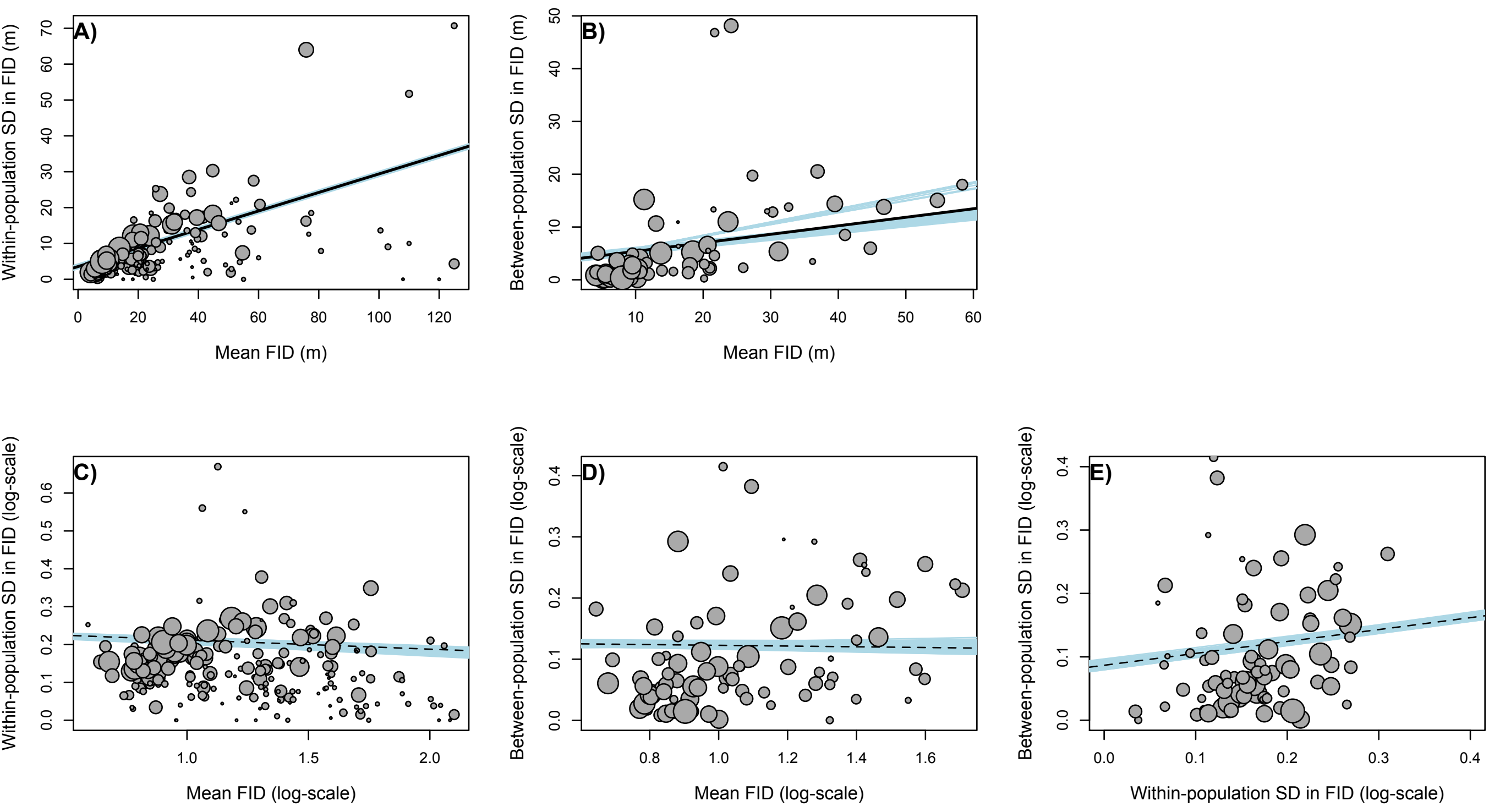
